\title{
Intestinal helminthiasis and urinary schistosomiasis in some villages of ljebu North, Ogun State, Nigeria
}

\author{
O. M. Agbolade ${ }^{1 *}$, D. O. Akinboye ${ }^{2}$ and A. Awolaja ${ }^{3}$ \\ ${ }^{1}$ Department of Biological Sciences, Olabisi Onabanjo University,P. M. B. 2002, Ago-Iwoye, Nigeria. \\ ${ }^{2}$ Department of Zoology,University of Ibadan, Ibadan, Nigeria. \\ ${ }^{3}$ Department of Primary Health Care, ljebu North Local Government,P. M. B. 1004, Ijebu-Igbo,Ogun State, Nigeria.
}

Accepted 31 December 2003

Intestinal helminthiasis and urinary schistosomiasis were studied between April and December, 2002, in six villages of ljebu North Local Government, Ogun State, Nigeria. Faecal samples from 199 subjects were examined using direct smear and brine concentration methods. Urine samples were tested for haematuria and proteinuria using diagnostic reagent strips. Three helminthic parasites were identified in the faecal samples; Ascaris lumbricoides (62.8\%), hookworm (16.6\%) and Schistosoma haematobium $(2.5 \%)$. None of the parasites was sex-dependent. $A$. lumbricoides had $\geq 50 \%$ prevalence in all the age groups. The more common mixed infection was $A$. lumbricoides and hookworm $(22.5 \%)$. The prevalences of haematuria and proteinuria were $7.5 \%$ and $15.7 \%$, respectively. After single dose levamisole treatment, $16.8 \%$ of the subjects with intestinal helminths voided and submitted $A$. lumbridoides adult worms.

Key words: Ascaris lumbricoides, hookworm, Schistosoma haematobium, haematuria, proteinuria, levamisole, Nigeria.

\section{INTRODUCTION}

Human intestinal helminths are among the most common infections occurring throughout the developing world. These infections have been associated with low standard of sanitation, and between 500 million and one billion people are estimated to be infected annually worldwide (Peters, 1970; WHO, 1987). There are several reports from various parts of Nigeria on human intestinal helminths, including those of Awogun et al. (1995), Nwaorgu et al. (1998), Taiwo and Agbolade (2000), and Adeyeba and Akinlabi (2002).

Urinary schistosomiasis due to Schistosoma haematobium infection is also endemic in many parts of Nigeria (WHO, 1993), and there are also several reports including those of Mafiana and Omotayo (1998), and Anosike et al. (2001).

To the best of our knowledge, no previous report on human intestinal helminthiasis and urinary schistosomiasis is known from rural communities of ljebu North, Ogun State, Nigeria. In view of the negative socio-

\footnotetext{
*Corresponding author. E-mail: agbolmos@yahoo.com.
}

economic impact of these parasitic infections on infected humans, efforts were made to elucidate their epidemiological stata. Treatment using levamisole (Ketrax) was also done.

\section{MATERIALS AND METHODS}

\section{Study area}

The study area consisted of Abule-Ige, Fanowo, Idi-Omo, Odo-Oye, Oke-Ogbe and Oniyangi villages in ljebu North Local Government Area of Ogun State, Nigeria. The villages range between $8 \mathrm{~km}$ and $15 \mathrm{~km}$ from Ago-Iwoye township (the main seat of Olabisi Onabanjo University). The study area is located between latitudes $6^{\circ} 57^{\prime}$ and $7^{\circ} 00^{\prime} \mathrm{N}$, longitudes $3^{\circ} 45^{\prime}$ and $3^{\circ} 48^{\prime} \mathrm{E}$.

The area is in the rain forest belt and the inhabitants are mainly subsistent farmers. There is one public primary school in each of Oke-Ogbe and Odo-Oye villages. There is one public health centre in the study area, which is located at Oke-Ogbe.

\section{Pre-survey protocols and questionnaire administration}

Prior to the commencement of the study, the head of each of the villages was contacted for enlightenment and permission. Through 


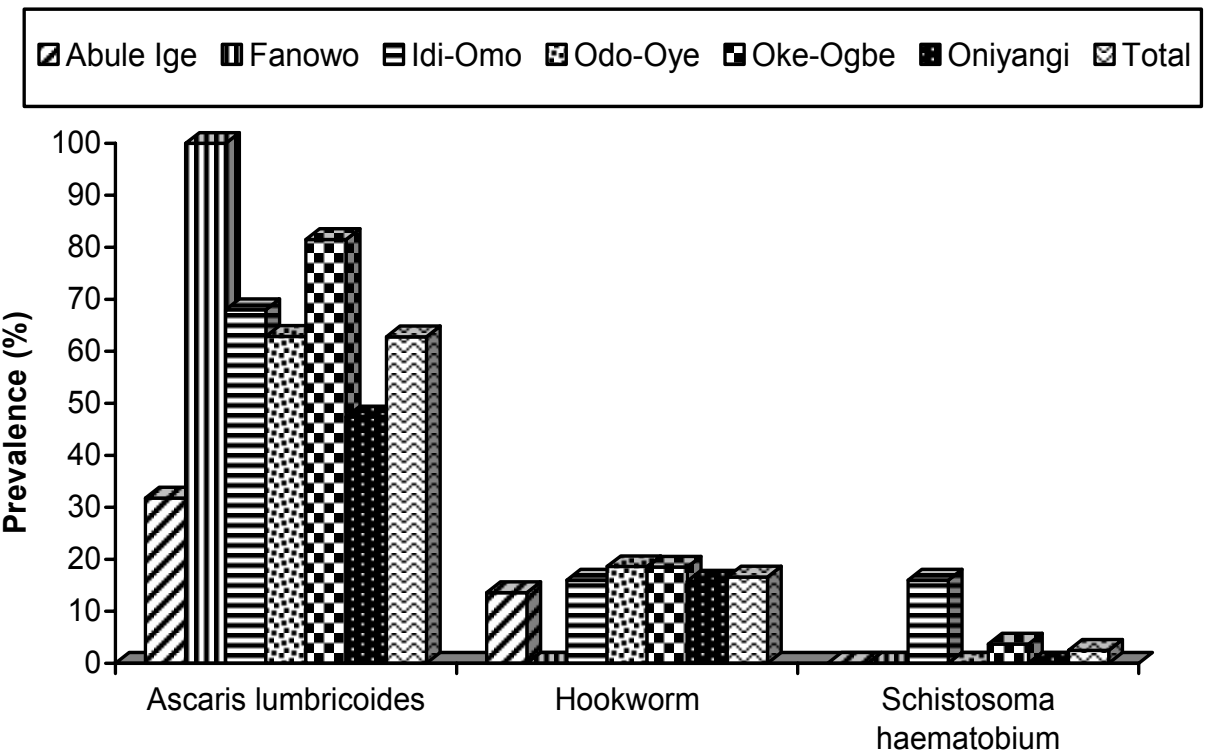

Parasitic Helminths

Figure 1. Prevalence of parasitic helminths according to village.

the assistance of the village heads, health workers and school teachers, the villagers were mobilized and encouraged to participate in the study. Nevertheless, only those who consented were eventually included in the study.

For each participating subject, a questionnaire was used to obtain information such as age, sex, source of drinking water, hygienic practices and history of presence of blood in stool (dysenteric syndrome).

\section{Collection and examination of samples}

Early morning faecal samples were collected from 199 subjects (males 79, females 120) between April and December 2002. In the laboratory each faecal sample was examined using direct faecal smear and brine concentration methods (Piekarski, 1989; WHO, 1991). For the brine concentration method, harvesting of helminth eggs was done using coverslip, which was left on the brine-faecal sample suspension for three to five minutes. All egg counts were expressed as eggs/g of faeces.

Urine samples were collected between the $11.00 \mathrm{~h}$ and $14.00 \mathrm{~h}$ in labelled specimen bottles. The samples were immediately tested for haematuria and proteinuria using diagnostic reagent strips.

\section{Levamisole treatment}

This was done with a single dose of 120 - $200 \mathrm{mg}$ levamisole (Ketrax). Each treated subject was requested to collect and keep any expelled worms in a specimen bottle containing $10 \%$ formalin solution.

\section{RESULTS}

Out of the 199 subjects examined, 129 (64.8\%) had helminthic infections. Three helminthic parasites were identified which were Ascaris lumbricoides, hookworm and S. haematobium. In Fanowo, only A. lumbricoides infection was recorded (Figure 1). In the remaining five villages, the prevalences of $A$. lumbricoides were statistically higher than those of hookworm $(P<0.05)$. $S$. haematobium ova were recorded in Idi-Omo $(16.7 \%)$ and Oke-Ogbe (3.7\%). In the total population, A. lumbricoides had significantly highest prevalence of $62.8 \%$, while $S$. haematobium had the least prevalence of $2.5 \%(\mathrm{P}<$ $0.05)$. The prevalence of hookworm was $16.6 \%$.

The total prevalence of $A$. lumbricoides among males, which was $65.8 \%$, was not significantly different $(P>$ 0.05 ) from the total prevalence among females, which was $60.8 \%$. Similarly, the total prevalence of hookworm among males, which was $15.2 \%$, was not statistically different $(P>0.05)$ from the total prevalence among females, which was $17.5 \%$. S. haematobium had equal total prevalences among males $(2.5 \%)$ and females $(2.5 \%)$. The observed age-sex-stratified prevalences of A. lumbricoides, hookworm and $S$. haematobium are shown in Figure 2. A. lumbricoides had high prevalences in all the age groups. Among males, the highest prevalence of $A$. lumbricoides $(100 \%)$ was recorded among $16-30$ years age groups, while the statistically highest prevalence $(91.7 \%)$ among females occurred in the $1-15$ years age group $(P<0.05)$. The prevalence of hookworm among the age groups were low compared with $A$. lumbricoides infection. In males, the prevalences of hookworm among $1-15$ and $31-45$ years age groups were statistically similar $(P>0.05)$.

Among the infected subjects, the prevalence of mixed infection due to $A$. lumbricoides and hookworm was $22.5 \%$. The prevalence of mixed infection due to $A$. lumbricoides and $S$. haematobium was $3.9 \%$. The 


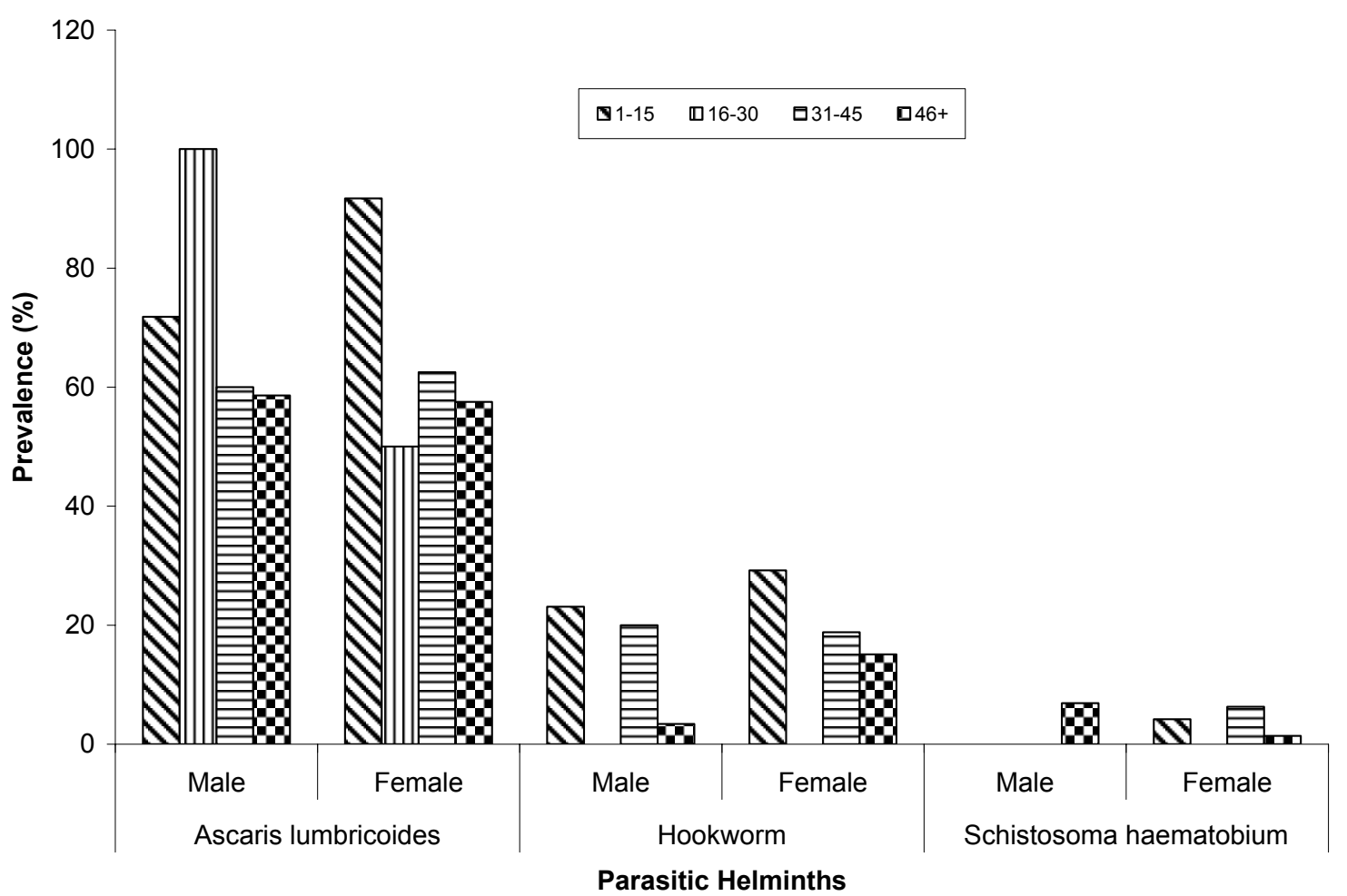

Figure 2. Prevalence of parasitic helminths according to sex and age.

intensity range of $A$. lumbricoides was $4,000-20,000$ eggs/g of faeces, while that of hookworm was 4,000 8,000 eggs/g of faeces. S. haematobium had an intensity of $>800$ eggs $/ g$ of faeces in each positive subject.

Questionnaire administration on the examined subjects revealed that all the villagers obtained their drinking water from wells and streams, and $99 \%$ of them practiced indiscriminate and open defaecation. $31.2 \%(62)$ of the subjects indulged in finger-nail nibbling. $17.6 \%$ (35) of the subjects had frequent stomach pain, while $7.5 \%$ (15) passed out blood with stool (dysenteric syndrome). Among those with dysenteric syndrome, 53.3\% had double infection with $A$. lumbricoides and hookworm.

Out of the 159 subjects whose urine samples were screened using diagnostic reagent strips, $12(7.5 \%)$ and $25(15.7 \%)$ had haematuria and proteinuria, respectively. $84.9 \%(135)$ of these subjects frequented ponds and streams for purposes including bathing, swimming, laundry and water-fetching for domestic purposes.

After levamisole treatment, $21(16.8 \%)$ of the subjects with intestinal helminths voided $A$. lumbridoides adult worms which were collected. The number of $A$. lumbricoides worms expelled ranged from one to seven worms per subject. Five subjects expelled approximately equal numbers of males and females. Six subjects passed out $A$. lumbricoides worm but did not collect them.

\section{DISCUSSION}

This study showed that $A$. lumbricoides and hookworm infections were possibly more widespread in the study area than $S$. haematobium infection. The higher prevalence $(62.8 \%)$ of $A$. lumbricoides than that of hookworm (16.6\%) agreed with some previous reports (Taiwo and Agbolade, 2000; Adeyeba and Akinlabi, 2002), but disagreed with that of Nwaorgu et al. (1998). The high prevalence of $A$. lumbricoides recorded in this study indicates high level of unhygienic practices among the villagers which enhanced transmission in the communities. This is corroborated by the fact that virtually all the villagers practiced indiscriminate and open defaecation while $31.2 \%$ indulged in finger-nail nibbing. Indiscriminate and open defaecation would also have enhanced the presence of hookworm infection in the study area.

The absence of Trichuris trichiura infection in the study area was unexpected since it is known that similar conditions generally influence its endemicity and that of A. lumbricoides infection (Ukoli, 1984). Possibly some false negatives were unavoidably recorded, or those having $T$. trichiura infection did not participate in the study. The observation of $S$. haematobium eggs in faeces in this study agreed with a previously established phenomenon (Smyth, 1996). Haematuria had been 
considered a reliable indicator of urinary schistosomiasis in south-west Nigeria (Mafiana and Omotayo, 1998). The presence of haematuria in many of the villagers examined possibly further demonstrates the endemicity of $S$. haematobium infection in the study area. Based on haematuria, the prevalence of $S$. haematobium in the study area might have been close to $7.5 \%$. Most of the villagers in the study area frequented ponds and streams and were thus exposed to $S$. haematobium infection.

In the present study, the three helminthic infections recorded were not sex-dependent in conformity with some reports (Agbolade and Odaibo, 1996; Mafiana and Omotayo, 1998; Taiwo and Agbolade, 2000). The $\geq 50 \%$ prevalence of $A$. lumbricoides infection in all the age groups suggests that the problems of unhygienic practices and low standard of sanitation were not restricted to any age group, in the study area.

Based on WHO (1987) classification, the intensity of $A$. lumbricoides in this study was moderate, while that of hookworm infection was heavy. The intensity of $S$. haematobium also appeared heavy (WHO, 1993). The more common double infection recorded was $A$. lumbricoides and hookworm. The presence of dysenteric syndrome in some of the subjects with double infection possibly indicates the severity of the combined influence of the two parasites.

Levamisole treatment of the examined subjects would have contributed positively to the control of $A$. lumbricoides and hookworm in the study area. Although no post-treatment faecal sample examination was done in this study, a related study showed $100 \%$ effectiveness of levamisole against $A$. lumbricoides and hookworm (Ogbe and Adu, 1990). It is hoped that the little antiintestinal helminth infections campaign done in the study area will remain with the villagers. Nevertheless, there is need for concerted periodical education and mass treatment to effectively control intestinal helminths and urinary schistosomiasis in the study area.

\section{ACKNOWLEDGEMENT}

We appreciate the field assistance of Mrs. Muritala, a village health worker at Oke-Ogbe Health Centre, Ogun State.

\section{REFERENCES}

Adeyeba OA, Akinlabi AM (2002). Intestinal parasitic infections among school children in a rural community, southwest Nigeria. Nig. J. Parasitol. 23:11-18.

Agbolade OM, Odaibo AB (1996). Schistosoma haematobium infection among pupils and snail intermediate hosts in Ago-Iwoye, Ogun State. Nig. J. Parasitol. 17:17-21.

Anosike JC, Nwoke BEB, Njoku AJ (2001). The validity of haematuria in the community diagnosis of urinary schistosomiasis infection. $\mathrm{J}$. Helminthol. 75:223-225.

Awogun IA, Okwerekwu FEO, Oyawoye OA, Bello AB (1995). Helminthic infections and anaemia among pregnant women attending antenatal clinic in llorin, Nigeria. Bioscience Res. Comm. 7:41-45.

Mafiana CF, Omotayo AB (1998). Urinary schistosomiasis: An evaluation of microscopic egg count and chemical reagent strip in children in south-west Nigeria. Helminthologia 35:31-35.

Nwaorgu OC, Okeibunor J, Madu E, Amazigo U, Onyegegbu N, Evans D (1998). A school-based schistosomiasis and intestinal helminthiasis control programme in Nigeria: acceptability to community members. Trop. Med. Int. Hlth 3:842-849.

Ogbe MG, Adu OO (1990). Intestinal helminthiasis in an orphanage in Nigeria. Bioscience Res. Comm. 2:105-118.

Peters W (1978). Medical aspects: Comments and discussions II. In: Taylor AER, Muller R. (eds.) The relevance of parasitology to human welfare today. Symposium of the British Society for Parasitology 16:25-40.

Piekarski G (1989). Medical parasitology. Springer-Verlag, Berlin

Smyth JD (1996). Animal parasitology. Cambridge University Press, Cambridge.

Taiwo AK, Agbolade OM (2000). Intestinal helmithiasis among school children in Oru, Ogun State, Nigeria. Nig. J. Sci. 34:283-286.

Ukoli FMA (1984). Introduction to parasitology in Tropical Africa. John Wiley and Sons Ltd., Chichester.

WHO (1987). Prevention and control of intestinal parasitic infections. WHO Technical Report Series, No. 749.

WHO (1991). Basic laboratory methods in medical parasitology. World Health Organisation Geneva.

WHO (1993). The control of schistosomiasis: second report of the Who Expert Committee WHO Technical Report Series, No. 830. 